\title{
Nontuberculous Mycobacteria Isolated from Tuberculosis Suspects in Ibadan, Nigeria
}

\author{
Simeon Idowu Cadmus, ${ }^{1}$ Bassirou Diarra, ${ }^{2}$ Brehima Traore, ${ }^{3}$ Mamoudou Maiga, \\ Sophia Siddiqui, ${ }^{2}$ Anatole Tounkara, ${ }^{2}$ Olutayo Falodun, ${ }^{4}$ Wole Lawal, ${ }^{5}$ \\ Isaac Folurunso Adewole, ${ }^{6}$ Rob Murphy, ${ }^{7}$ Dick van Soolingen, ${ }^{8}$ and Babafemi Taiwo ${ }^{7}$ \\ ${ }^{1}$ Tuberculosis and Brucellosis Research Laboratories, Department of Veterinary Public Health \& Preventive Medicine, \\ University of Ibadan, Ibadan 200005, Nigeria \\ ${ }^{2}$ Project SEREFO (Centre de Recherche et de Formation sur le VIH/Sida et la Tuberculose)/University of Sciences, \\ Technics and Technologies of Bamako (USTTB), Bamako, Mali \\ ${ }^{3}$ Centre d'Infectiologie Charles Mérieux, rue Dr. Charles Mérieux, ex-base aérienne, BP E2283, Bamako, Mali \\ ${ }^{4}$ Department of Microbiology, University of Ibadan, Ibadan 200005, Nigeria \\ ${ }^{5}$ Tuberculosis and Leprosy Division, Oyo State Ministry of Health, Ibadan 200005, Nigeria \\ ${ }^{6}$ Department of Obstetrics and Gynaecology, University College Hospital, Ibadan 200005, Nigeria \\ ${ }^{7}$ Division of Infectious Disease and Center for Global Health, Northwestern University, 645 North Michigan Avenue, \\ Chicago, IL 60611, USA \\ ${ }^{8}$ Diagnostic Laboratory for Bacteriology and Parasitology (BPD), Center for Infectious Disease Research, \\ Diagnostics and Perinatal Screening (IDS), National Institute for Public Health and the Environment (RIVM), \\ P.O. Box 1, 3720 BA Bilthoven, Netherlands
}

Correspondence should be addressed to Simeon Idowu Cadmus; sibcadmus@yahoo.com

Received 31 October 2015; Accepted 6 March 2016

Academic Editor: Abhineet S. Sheoran

Copyright (C) 2016 Simeon Idowu Cadmus et al. This is an open access article distributed under the Creative Commons Attribution License, which permits unrestricted use, distribution, and reproduction in any medium, provided the original work is properly cited.

In Nigeria, one of the highest tuberculosis (TB) burdened nations, sputum smear microscopy is routinely employed for TB diagnosis at Directly Observed Treatment Short-Course (DOTS) Centers. This diagnostic algorithm does not differentiate Mycobacterium tuberculosis complex (MTC) from nontuberculous mycobacteria (NTM). Between December 2008 and January 2009, consecutive patients diagnosed with TB were screened for inclusion at 10 DOTS centers in Ibadan, Nigeria. To verify Mycobacterium species in patients diagnosed, we cultured and identified mycobacterial isolates using PCR, line probe assay, and spoligotyping techniques. From 48 patients screened, 23 met the inclusion criteria for the study. All the 23 study patients had a positive culture. Overall, we identified 11/23 patients (48\%) with MTC only, 9/23 (39\%) with NTM only, and 3/23 (13\%) with evidence of both MTC and NTM. Strains of MTC identified were Latin American Mediterranean (LAM) genotype $(n=12)$, M. africanum $(n=1)$, and the genotype family T $(n=1)$. Four M. avium-intracellulare-M. scrofulaceum complexes, one M. chelonae complex, one M. abscessus, and one M. intracellulare were identified. Our findings underscore the need to incorporate molecular techniques for more precise diagnosis of TB at DOTS centers to improve clinical outcomes and safe guard public health, particularly in TB endemic countries.

\section{Introduction}

For several years, Nigeria has remained in the league of the highest TB burdened nations of the world and is currently ranked 4th globally [1]. Sputum smear microscopy is routinely employed for TB diagnosis at Directly Observed
Treatment Short-Course (DOTS) Centers in Nigeria [2]. The diagnostic algorithm entails interpreting presence of acid fast bacilli in sputum smear microscopy as TB. This algorithm does not differentiate Mycobacterium tuberculosis complex (MTC) from nontuberculous mycobacteria (NTM) [3], which are ubiquitous environmental mycobacteria [4-6], 
TABLE 1: Epidemiological profile of patients with positive mycobacterial culture.

\begin{tabular}{|c|c|c|c|c|c|c|}
\hline Number of patients & $\begin{array}{l}\text { Clinical } \\
\text { history }\end{array}$ & $\begin{array}{l}\text { Ziehl- } \\
\text { Neelsen } \\
\text { staining }\end{array}$ & $\begin{array}{c}\text { Culture on selective agar } \\
\text { MTC/NTM } \\
\text { (phenotypic speciation) }\end{array}$ & $\begin{array}{l}\text { INNO-LiPA test } \\
\text { NTM (molecular } \\
\text { speciation) }\end{array}$ & $\begin{array}{l}\text { Spoligotyping } \\
\text { MTC } \\
\text { (molecular } \\
\text { speciation) }\end{array}$ & $\begin{array}{l}\text { Identity of } \\
\text { patients }\end{array}$ \\
\hline 10 & New & + & M. tuberculosis & NR & + & $\begin{array}{c}1,3,4,6,7,8 \\
10,12,13,14\end{array}$ \\
\hline 1 & Relapse & + & M. tuberculosis & NR & + & 5 \\
\hline 1 & New & + & $\begin{array}{c}\text { M. tuberculosis } \\
\text { Unclassified NTM }\end{array}$ & MAIS complex & + & 2 \\
\hline 1 & Relapse & + & $\begin{array}{l}\text { M. tuberculosis } \\
\text { Unclassified NTM }\end{array}$ & $\begin{array}{l}\text { MAIS complex } \\
\text { M. intracellulare }\end{array}$ & + & 11 \\
\hline 1 & New & + & $\begin{array}{l}\text { M. tuberculosis } \\
\text { Unclassified NTM }\end{array}$ & ND & + & 9 \\
\hline 6 & New & + & Unclassified NTM & ND & NR & $\begin{array}{c}15,16,17,18 \\
19,21\end{array}$ \\
\hline 1 & New & + & Unclassified NTM & $\begin{array}{l}\text { MAIS complex } \\
\text { M. chelonae } \\
\text { complex }\end{array}$ & NR & 20 \\
\hline 1 & New & + & Unclassified NTM & $\begin{array}{l}\text { MAIS complex } \\
\text { M. abscessus }\end{array}$ & NR & 22 \\
\hline $1^{*}$ & Relapse & - & Unclassified NTM & MAIS complex & NR & 23 \\
\hline
\end{tabular}

* refers to patient with negative sputum smear but positive X-ray; +/- positive or negative; NR: not required; ND: not determined.

MAIS complex: Mycobacterium avium-intracellulare-Mycobacterium scrofulaceum complex.

increasingly isolated from immune competent and immune compromised hosts $[2,7,8]$. Also other acid fast bacilli, like Rhodococcus spp. cannot be identified using this approach. Since clinical implications and therapeutic options for NTM differ markedly from those for $\mathrm{TB}$, accurate discrimination of TB from NTM infection is essential to avoid underor overtreatment of either condition, bearing in mind the potential patient care and economic consequences $[6,8]$.

To explore whether misdiagnosis is a problem in Nigeria, we cultured sputum and conducted molecular characterization of acid fast isolates in patients already diagnosed with TB based on the local diagnostic algorithm at DOTS centers in Ibadan, Southwestern Nigeria.

\section{Materials and Methods}

2.1. Patient Cohort. This study was conducted at 10 DOTS centers in Ibadan, Southwestern Nigeria, between December 2008 and January 2009. Consecutive patients diagnosed with TB (based on positive smear microscopy or symptoms plus chest X-ray) were screened for inclusion. Those on TB therapy for more than 3 days were excluded. Demographic, clinical, and radiologic data of eligible participants were extracted from medical records. Ethical clearance for the study was obtained from Oyo State Ministry of Health Ethical Review Board as part of an ongoing TB project.

2.2. Bacteria Culture and Morphological Identification. Sputum sample from each patient was digested and decontaminated with $\mathrm{N}$-acetyl-L-cysteine, $4 \% \mathrm{NaOH}$; concentrated by high speed centrifugation; stained with auramine-rhodamine; and examined by fluorescent microscopy. Pellets from each sample were cultured simultaneously on Mycobacteria growth indicator (MGIT) tubes containing oleic acid-albumin-dextrose-catalase and polymyxin-amphotericin B-nalidixic acid-trimethoprim-azlocillin and Middlebrook 7H11 agar medium plates (for morphological identification). The inoculated media were incubated at $37^{\circ} \mathrm{C}$ with $7 \% \mathrm{CO}_{2}$ for 6 weeks as previously described [9]. Positive cultures were screened by Ziehl-Neelsen and Gram stain in order to exclude other positive Gram staining bacteria. Observation of colony morphology, recognition of mixed infections (more than one Mycobacterium species), and differentiation of the patients' cultures into MTC and NTM was carried out through microscopic examination of the growths on the Middlebrook 7H11 culture plates [5].

2.3. Molecular Identification. Definitive molecular identification for NTM was carried out using mainly the INNOLiPA assay (INNO-LiPA Mycobacteria v2; Innogenetics ${ }^{\circledR}$ N.V., Gent, Belgium) for the amplification of the 16S-23S rRNA. Only five patients' samples that were morphologically identified as NTM were available for this. The spoligotyping technique was carried out using a commercially available kit (Spoligotyping Isogen Life Science, De Meern, Netherlands) and this was used mainly for MTC (for all morphologically identified MTC samples).

\section{Results and Discussion}

A total of 48 patients were screened for inclusion. Out of these, 25 patients had received TB therapy for more than 


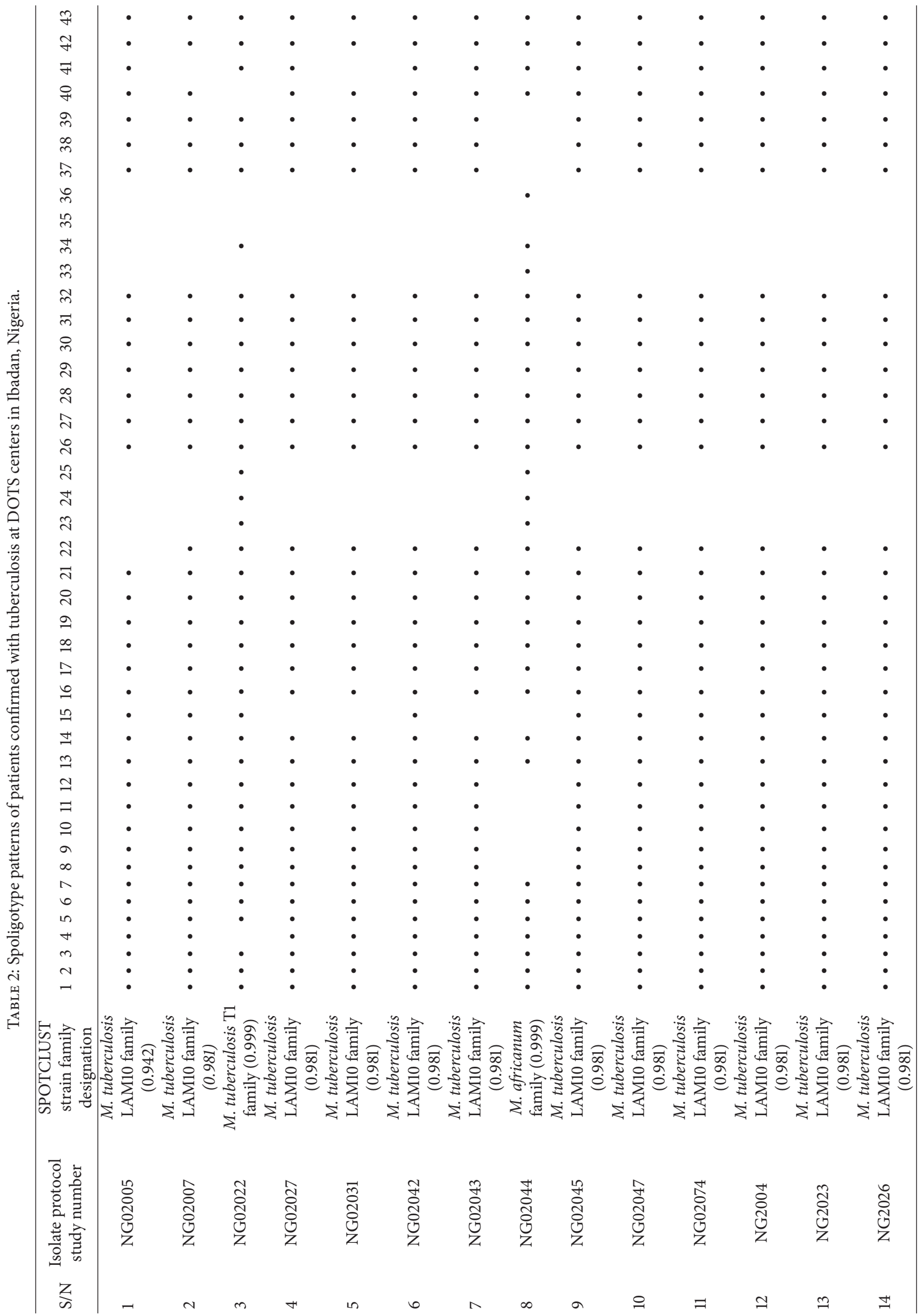


three days and were excluded. Thus, the study population comprised 23 patients ( 3 diagnosed as TB relapse due to prior full course of TB therapy and 20 diagnosed as new TB cases). Twenty-two of these patients had been diagnosed with TB based on positive smear microscopy while one patient had negative microscopy result but positive chest X-ray (Table 1). All the 23 study patients had a positive culture. Based on morphological growths on Middlebrook 7H11 agar culture plates and the molecular analyses, we identified $11 / 23$ patients (48\%) with MTC only, 9/23 (39\%) with NTM only, and 3/23 (13\%) with evidence of both MTC and NTM bacteria present (Table 1). The strains of MTC identified through the spoligotyping represented Latin America Mediterranean (LAM) genotype $(n=12), M$. africanum $(n=1)$, and the genotype family $\mathrm{T}(n=1)$ (Table 2). Using the INNO-LiPA identification assay, four $M$. avium-intracellulare-M. scrofulaceum complexes, one $M$. chelonae complex, one M. abscessus, and one M. intracellulare were identified (Table 1). Seven NTM strains identified by colony morphology on Middlebrook 7H11 solid media were unavailable for the INNO-LiPA assay. In all, we identified 28 strains of Mycobacteria (i.e., both M. tuberculosis complex and NTM) from the 23 patients screened.

In this study, NTM was identified in 50\% (14/28) of mycobacterial isolates recovered from the 23 patients diagnosed with TB at DOTS centers in Ibadan. Critically, using culture, only NTM was recovered from $40 \%(8 / 20)$ of patients diagnosed as new TB cases and in 33\% (1/3) of patients diagnosed as relapsed TB (i.e., among new patients, $40 \%$ of them had only NTM without mixed infection with $M$. tuberculosis and among relapse patients only one of them had single infection with NTM without coinfection with $M$. tuberculosis). This is an important finding since the potential public health implications of TB misdiagnosis in patients infected with NTM only are far-reaching. Invariably, these patients are treated with anti-TB drugs including scarce and more costly second line drugs in those with presumed relapse and multidrug-resistant tuberculosis (MDR-TB). Associated toxicities such as isoniazid hepatotoxicity may be lifethreatening while symptomatic NTM remains untreated. An essential component of NTM treatment (macrolide) is not included in any TB regimen. In addition, the public health estimates of TB and MDR-TB are correspondingly distorted by these misdiagnoses coupled with unnecessary expenditure of healthcare resources [6].

The findings of this study should be interpreted in the context of its limitations which include evaluation of only a single sputum sample from each patient $[3,7]$. Repeated isolation of the same NTM would have provided more definite evidence of true NTM infections. Risk factors associated with NTM pulmonary disease were not assessed comprehensively due to limited clinical evaluations at the DOTS centers. Specifically, information on HIV serological status was available in only a minority of patients; however the 2008 prevalence data for the study area was only $2.2 \%$ [10]. This is relevant since HIV affects the clinical manifestation of TB and can increase pulmonary colonization with NTM [6]. Finally, there was nonavailability of seven NTM isolates for molecular characterization using the INNO-LiPA assay.

\section{Conclusions}

Despite the limitations of our findings, this study indicates the possibility of erroneous TB diagnosis and treatment in patients infected with NTM in our population. Ongoing studies such as the national MDR-TB survey will shed more light on the scope of this problem in Nigeria. Our findings underscore an urgent need to incorporate molecular techniques for more precise diagnosis of TB at DOTS centers to improve clinical outcomes and safeguard public health, particularly in TB endemic countries.

\section{Competing Interests}

The authors have declared no competing interests.

\section{Acknowledgments}

The authors thank Baiba Berzins of the Division of Infectious Diseases, Northwestern University Feinberg School of Medicine, Chicago, IL, USA, for facilitating all the logistic aspects of this work. Special thanks also goes to the entire team of the National Tuberculosis Reference Laboratory, National Institute for Public Health and the Environment, Bilthoven, Netherlands, for assisting with part of the laboratory analysis. Most importantly, the authors appreciation goes to the patients at the DOTS centers for their cooperation in making this project possible. The project described was partly funded by Grant no. D43TW007995 from the Fogarty International Center. The content is solely the responsibility of the authors and does not necessarily represent the official views of the Fogarty International Center or the National Institutes of Health. Finally, The authors want to specially acknowledge Professor Tounkara Anatole (one of the authors) who died before submission of this paper.

\section{References}

[1] WHO, Global Tuberculosis Report 2015, World Health Organization, 20th edition, 2015, http://apps.who.int/iris/bitstream/ 10665/191102/1/9789241565059_eng.pdf?ua=1.

[2] WHO, Global Tuberculosis Control: Epidemiology, Strategy, Financing: WHO Report 2009, 2009, http://www.afro.who.int/ index.php?option $=$ com_docman\&task=doc_download\&gid $=$ 2821.

[3] The American Thoracic Society, "Diagnostic standards and classification of tuberculosis in adults and children," American Journal of Respiratory and Critical Care Medicine, vol. 161, no. 4, pp. 1376-1395, 2000.

[4] S. M. Arend, D. van Soolingen, and T. H. M. Ottenhoff, "Diagnosis and treatment of lung infection with nontuberculous mycobacteria," Current Opinion in Pulmonary Medicine, vol. 15, no. 3, pp. 201-208, 2009.

[5] D. E. Griffith, T. Aksamit, B. A. Brown-Elliott et al., "An official ATS/IDSA statement: diagnosis, treatment, and prevention of nontuberculous mycobacterial diseases," American Journal of Respiratory and Critical Care Medicine, vol. 175, no. 4, pp. 367416, 2007.

[6] B. Taiwo and J. Glassroth, "Nontuberculous mycobacterial lung diseases," Infectious Disease Clinics of North America, vol. 24, no. 3, pp. 769-789, 2010. 
[7] H. Grubek-Jaworska, R. Walkiewicz, A. Safianowska et al., "Nontuberculous mycobacterial infections among patients suspected of pulmonary tuberculosis," European Journal of Clinical Microbiology and Infectious Diseases, vol. 28, no. 7, pp. 739-744, 2009.

[8] P. Tabarsi, P. Baghaei, P. Farnia et al., "Nontuberculous mycobacteria among patients who are suspected for multidrugresistant tuberculosis-need for earlier identification of nontuberculosis mycobacteria," The American Journal of the Medical Sciences, vol. 337, no. 3, pp. 182-184, 2009.

[9] B. Diarra, S. Siddiqui, D. Sogoba et al., "Mycobacterium tuberculosis Beijing Strain, Bamako, Mali," Emerging Infectious Diseases, vol. 16, no. 2, pp. 362-363, 2010.

[10] Federal Ministry of Health, "National HIV sero-prevalence sentinel survey among pregnant women attending antenatal clinics in Nigeria," Tech. Rep., Federal Ministry of Healt, Abuja, Nigeria, 2008. 


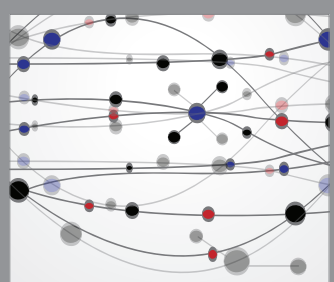

The Scientific World Journal
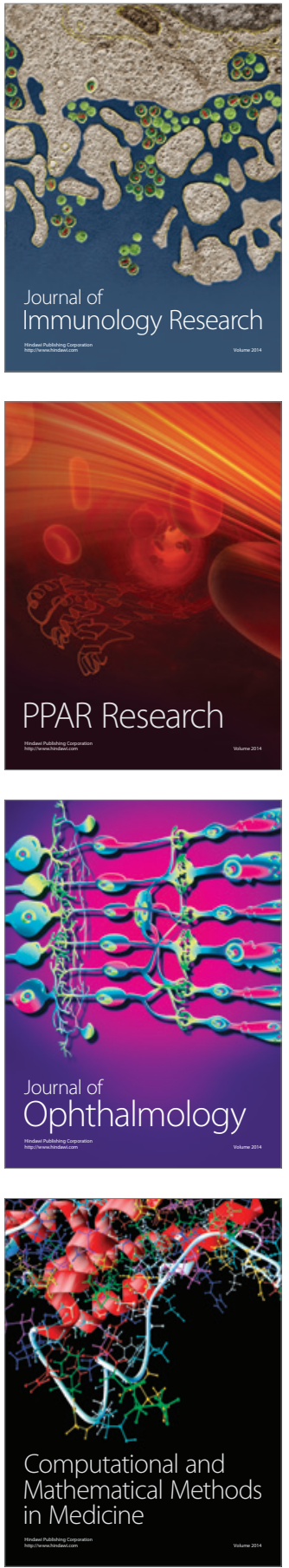

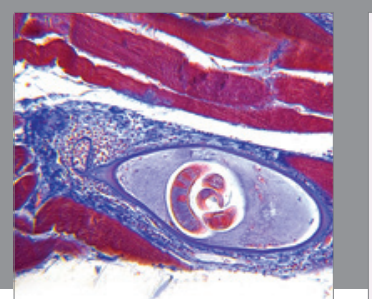

Gastroenterology Research and Practice

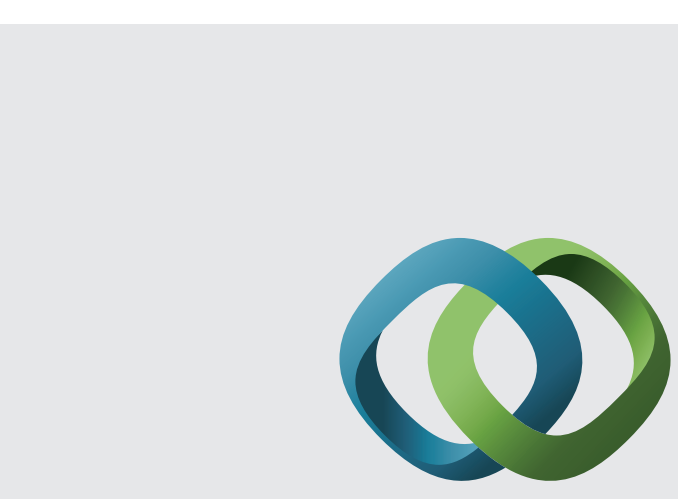

\section{Hindawi}

Submit your manuscripts at

http://www.hindawi.com
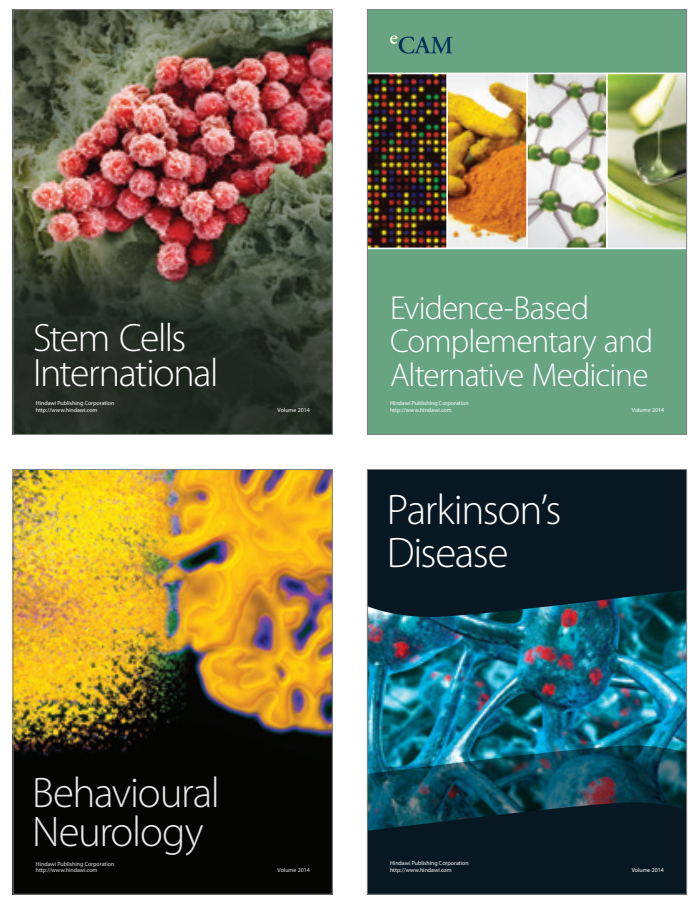
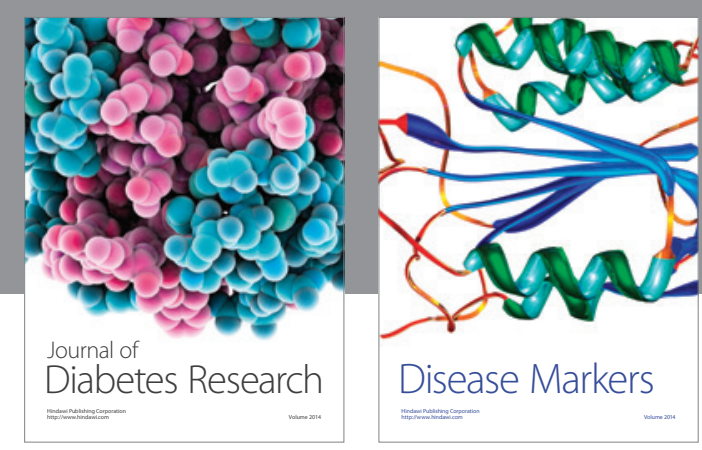

Disease Markers
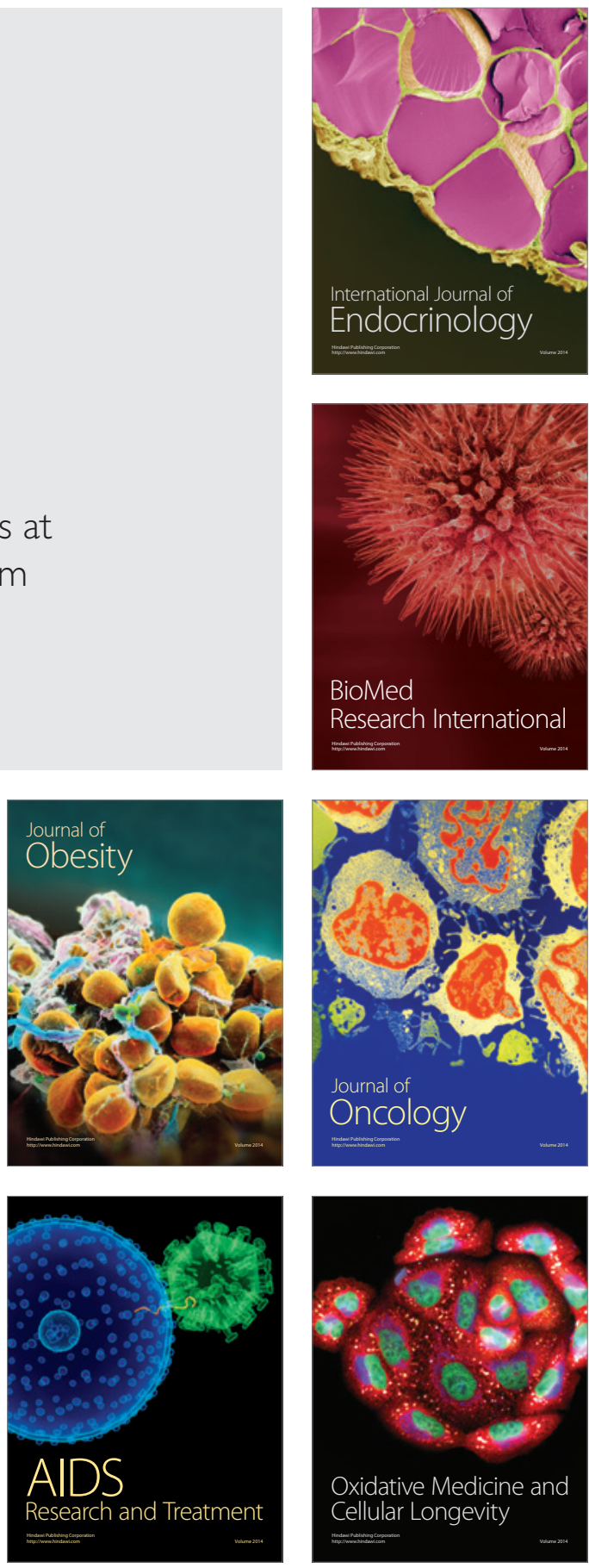